\title{
Estructura de la comunidad polinizadora en un cultivo de tomate Lycopersicon esculentum Mill (Solanaceae) y Análisis Multivariado de la calidad del fruto, Caldas - Colombia
}

\author{
Pollinators community structure and multivariant analysis of tomato's quality fruit \\ Lycopersicon esculentum Mill (Solanaceae) in a crop located in Caldas - Colombia.
}

\author{
Sandra Milena arias restrepo ${ }^{1}$, Jhon Deiber Torres Carrera ${ }^{1}$, Ligia Janneth Molina Rico ${ }^{1}$ \\ ${ }^{1}$ Licenciatura en biología y ciencias ambientales, Universidad del Quindío, Quindio, Colombia. \\ sandra.ariaseutp.edu.co \\ jhondeiber20@yahoo.es \\ janneth@uniquindio.edu.co
}

\begin{abstract}
Resumen- Se determinó la comunidad de polinizadores y se analizó la calidad del fruto de tomate Lycopersicon esculentum Mill, en Viterbo-Caldas. Se realizaron recorridos aleatorios en un semi-invernadero en floración con alta actividad pecoreadora registrando visitantes en plantas censadas y control, obtuvimos valores de abundancia y géneros por conteo en rangos de horas. Para la calidad del fruto se sometieron a un Análisis de Componentes Principales variables ecológicas y económicas. Se aplicó una prueba $T$, diversidad y una curva de acumulación de géneros; la representatividad de la muestrea se estimó con Chao1, ACE, ICE, Singletons y Doubletons. La polinización del cultivo fue de $\mathbf{5 7 . 7 \%}$. Determinamos cuatro géneros de abejas polinizadoras: Apis, Melipona, Agapostemon y Euglossa. Mayor actividad pecoreadora de 8 a 10 am. El peso y diámetro polar, se asociaron con Agapostemon $(\mathrm{R}=1, \mathrm{p}=\mathbf{0 . 0 0 2 6}, \mathrm{CP1}=\mathbf{7 8 . 3 \%} \mathrm{y}$ $\mathrm{CP2}=\mathbf{2 1 . 7 \%}$ ). La prueba $\mathrm{T}$, mostró diferencias del peso entre polinización cruzada $y$ autógama $(\mathrm{p}=\mathbf{0 . 0 0 3 5})$. La riqueza, dominancia y diversidad de polinizadores fue baja $(E=0.90460$, $\mathrm{DMg}=1.13677$, Dsp=0.25275, H'=1.25404). El esfuerzo de muestreo fue representativo con Chao $1100 \%$ y ACE $90 \%$. Aproximadamente el $50 \%$ del cultivo no es aprovechado productivamente. La polinización cruzada realizada por Agapostemon, mejora el peso y diámetro polar del fruto.
\end{abstract}

Palabras clave - Comunidad polinizadora, calidad del fruto, estadística multivariada, Lycopersicon esculentum, polinización cruzada.

Abstract - We determined the community pollinators and analyzed tomato's quality fruit Lycopersicon esculentum Mill cultivated in Viterbo-Caldas Colombia's municipality. We sampled in a flowering semi greenhouse doing randomly for highest foraging areas around. Were recorded plant-flower visitors each inspected plant and its control, obtaining abundance and genera values from those by counting per hour ranges. We used a Principal Component Analysis to analyze ecological and economical variables of fruit quality. We used a $T$, diversity. And genera accumulation curve was graphed. To analyze the sample representativeness we obtained the Chao1, ACE, ICE, Singletons y Doubletons estimators. Pollination percentage in crop $57.7 \%$. We taxonomically identified four genera of pollinating bees: Apis , Melipona, Agapostemon, and Euglossa. Highest foraging activity ranging from 8 to10 am was found. Weight vs polar diameter was close to Agapostemon $(R=1$, $\mathrm{p}=\mathbf{0 . 0 0 2 6}, \mathrm{CP} 1=\mathbf{7 8 . 3 \%}$ y $\mathrm{CP} 2=\mathbf{2 1 . 7 \%}$ ). $\mathrm{T}$-test showed significantly differences $(p=0.0035)$ between cross and self pollination. Diversity indices $(E=0.90460)$ suggested a low pollinators' richness, dominance and diversity $(\mathrm{DMg}=1.13677$; $\mathrm{Dsp}=0.25275$; $\left.H^{\prime}=1.25404\right)$. The sampling was representativeness as Chao 1 $100 \%$ and ACE $90 \%$ estimated. Among 50\% of the crop is not exploited productively. cross pollination makes by Agapostemon becomes fruits' weight and polar diameter improve.

Key Words - Cross pollination, fruit's quality characteristics, Lycopersicon esculentum, multivariate data analysis, pollinators community.

\section{INTRODUCCIÓN}

El desarrollo de plantas cultivadas en condiciones de semiinvernadero como el tomate Lycopersicon esculentum depende de factores como la temperatura, el suministro de nutrientes, agua e iluminación [1]. Dichos factores pueden ser controlados y optimizados en estas condiciones. Pese a ello, el desarrollo de estos sistemas se ve limitado tanto por razones económicas, como por el poco conocimiento de las implicaciones de un adecuado proceso de fecundación y sus efectos en la producción [1]. El sistema de producción de tomate bajo condiciones controladas, es relativamente nuevo en Colombia, lo que ha generado impactos significativos en extensiones de cultivo, productividad, rentabilidad y calidad del producto [2]. Así, un crecimiento en la expansión y producción de los cultivos, aumenta la demanda de la polinización, por lo que, en la obtención de frutos más grandes y atractivos comercialmente, se han utilizado métodos como fitohormonas, vibración manual y el uso de insectos polinizadores [3]. 
En el último método, se utilizan abejas para el mejoramiento de la polinización, fecundación, producción de semillas, los cuales, repercuten en la calidad del fruto. Sin embargo, se debe tener en cuenta que la eficiencia de polinización de un visitante floral, está estrechamente relacionada con la biología floral y el comportamiento de forrajeo del polinizador, los cuales toman néctar, polen, esencias y aceites; sin embargo, no todo visitante floral es un polinizador eficiente [Free, 1970 por [4]. Por lo anterior, la presencia de abejas en un cultivo permite que el polen se disperse con mayor facilidad, no causando algún tipo de daño físico a la planta, lo que converge en la producción de mejores cosechas [Alderz, 1996; Amaral et al., 1983, Connor, 1969; Free, 1986 por [5].

La polinización de plantas, cuyos frutos son de valor comercial dependen mayoritariamente de las abejas para su polinización, o ellas influyen en más del $80 \%$ de los casos. Esto hace de la polinización el principal aporte económico que las abejas ofrecen ya que incrementa la productividad de forma significativa [6].

En razón a lo anterior, el presente estudio tiene como fin determinar la estructura de la comunidad de polinizadores en un cultivo de tomate Lycopersicon esculentum Mill, de la variedad comercial Larga vida - cumanday en condiciones de semi-invernadero en el departamento de Caldas. Asimismo, se presenta un estudio de las relaciones entre polinizadores y características de la calidad del fruto basado en análisis multivariante.

\section{METODOLOGÍA}

\section{A. Área de estudio}

La zona de estudio se encuentra ubicada en el municipio de Viterbo en el departamento de Caldas (Figura 1), específicamente en la vereda el Socorro finca el Jardín, ubicada a $6.1 \mathrm{~km}$ de la cabecera municipal. Esta vereda se encuentra inmersa en una matriz que comprende cultivos de frutales, plátano, café y explotación de ganadería intensiva de carne y leche; piscicultura y porcicultura. Posee una temperatura promedio de $22^{\circ} \mathrm{C}$ y una altura de 1077 m.s.n.m. El punto de muestreo se localizó en las coordenadas geográficas N $05^{\circ} 05^{\prime} 943^{\prime}$ 'W $075^{\circ} 53^{\prime} 649^{\prime}$ '. El muestreo se realizó entre los meses de Agosto - Octubre de 2012.

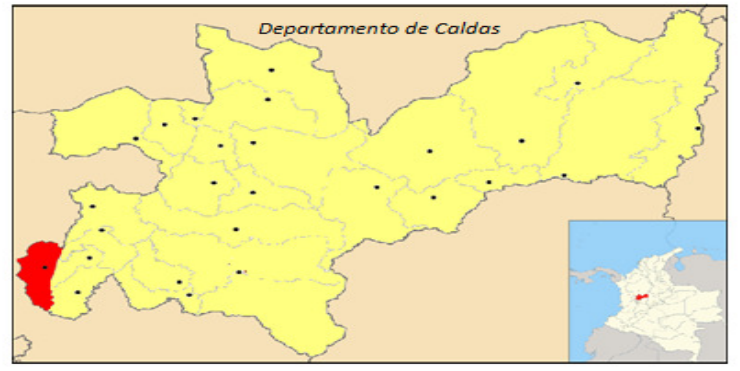

Figura 1. Sitio de estudio. Municipio de Viterbo (rojo) en el departamento de Caldas. Tomado de www.wikipedia.org

\section{B. Diseño de muestreo y toma de datos}

Previo a la toma de datos, se realizó un diagnóstico que consistió en estimar el porcentaje de polinización actual del cultivo. Esto se efectuó de la siguiente manera: se seleccionó al azar una calle y dentro de ésta, se escogieron aleatoriamente 100 individuos. De cada individuo, se seleccionó un racimo en antesis floral, es decir, que estuviese en estado de floración. De estos se realizó un conteo del número de flores pertenecientes a ese racimo. Una vez identificado el racimo en floración, se marcó con una cinta para su posterior revisión. De esta manera, ocho días después se ejecutó un conteo de la fructificación a través del número de frutos formados por cada flor registrada.

\section{Efectividad de visitantes florales}

Se seleccionó un semi-invernadero (sección 3), ya que las otras dos secciones no se encontraban en estado de floración. El cultivo para la producción del tomate (Lycopersicon esculentum Mill) se encuentra dispuesto en condiciones de semi-invernadero, las cuales se caracterizan por estructuras de capuchas con flujo de aire permanente como se muestra en la figura 2a. Con aproximadamente 4000 plantas, el cultivo se encuentra dispuesto en calles con individuos separados uno del otro a $30 \mathrm{~cm}$ de distancia.

Para el muestreo, se hicieron recorridos aleatorios permanentes donde se registraron los visitantes florales presentes en zonas de mayor actividad pecoreadora. Dichas zonas, se identificaron a través de detección por zumbido, el cual identifica a estos insectos.

Durante la toma de datos, cada planta censada fue tomada como unidad de muestreo. Se registró el número de flores visitadas por racimo y el insecto visitante. Este último fue enumerado como morfotipo. Una vez identificada la flor y el visitante, se procedió a embolsar el racimo floral, con bolsa de velo suizo impidiendo el contacto de otro visitante con el estigma de la flor (Figura 2b). Del mismo modo, la flor visitada fue marcada con una cinta de reconocimiento. Así, cada planta censada fue etiquetada con los datos de: fecha, número de planta, número de racimo y número de flores polinizadas por racimo.

Previo a los recorridos en el muestreo, se seleccionó asimismo una planta control dentro de la cual se escogió al azar un racimo antes de la antesis floral. Este también fue cubierto con velo suizo para así asegurar la polinización autógama. En los 10 transectos y con ayuda de una red entomológica con diámetro de $10 \mathrm{~cm}$, se capturaron las abejas que se encontraban visitando las flores del tomate, una vez recolectadas se procedió al sacrificio de éstas utilizando acetato de etilo. 


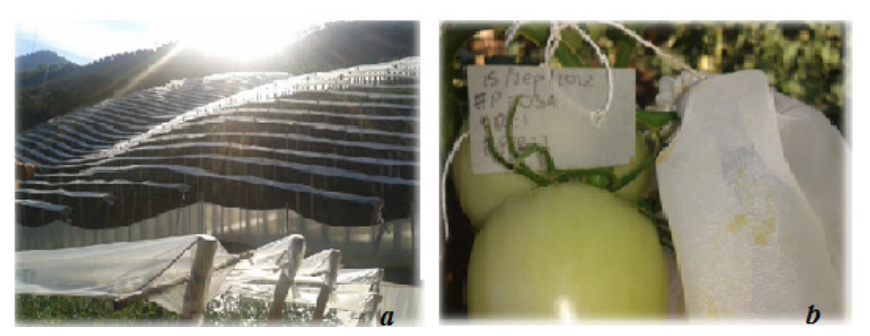

Figura 2. a) Semi-invernadero del cultivo de tomate, capuchas con aireación permanente. b) Etiqueta con numeración consecutiva, información del racimo floral y bolsa de velo suizo aislante de otras posibles visitas en las flores registradas.

2. Análisis de abundancia relativa, diversidad y esfuerzo de muestreo

Para el análisis de abundancia relativa de los géneros de visitantes florales se seleccionaron diez calles separadas, cada una por una calle, dentro de las cuales se escogieron al azar para cada una, 10 transectos de $20 \mathrm{~m}$ de longitud. Estos transectos se enumeraron de extremo a extremo de manera consecutiva, para así evitar el reconteo del mismo individuo dentro de cada transecto, realizándose así recorridos a lo largo de estos 20 metros en la misma dirección. Se registró el número de individuos previamente identificados mediante conteo directo a lo largo de cada transecto. Los recorridos se realizaron en tres rangos de horas establecidas: horario 1) 8:00-10:00 am, 2) 11:00-1:00pm y 3) 2:00-4:00 pm. La información de las abundancias (N) y los géneros identificados (S), fue útil para la estimación de los índices de diversidad. Asimismo, los valores $\mathrm{S}$ y $\mathrm{N}$, se utilizaron para graficar una curva de acumulación de géneros a lo largo de los 10 transectos para conocer el esfuerzo de muestreo. Esto se contrastó en detalle con la aplicación de los estimadores Chao1, ACE, ICE, Singletons y Doubletons.

\section{Calidad del fruto}

Utilizando los datos de la efectividad de la visita en las zonas de mayor actividad pecoreadora, se verificó la formación del fruto ocho días después donde cada racimo fue despojado de la cubierta. A cada fruto formado, se le realizó un seguimiento hasta su madurez. Una vez en este estado, los frutos se cosecharon y se sometieron a una toma de las siguientes variables morfométricas de interés económico: Peso (g), diámetro ecuatorial y diámetro polar $(\mathrm{cm})$. El peso fue tomado con una balanza electrónica y las medidas morfométricas con un calibrador. Asimismo, se tomó la variable numero de semillas por fruto, como una variable de interés ecológico. Dichas variables fueron utilizadas para realizar la comparación con la planta control.

\section{Análisis de datos}

Utilizando el programa EXCEL 2007, se estimó el porcentaje de polinización a partir del número de frutos formados desde el conteo total de flores en los racimos seleccionados. Además, se realizó un conteo manual a partir de valores absolutos del número de frutos clasificados a través de variables cualitativas, estas fueron representadas por condiciones finales del fruto producto de la polinización efectiva.

1. Abundancia relativa, diversidad y esfuerzo de muestreo

Asimismo usando el programa InfoStat versión 2012 [7], se realizó un gráfico de sectores para la frecuencia relativa utilizando las abundancias de los géneros en todos los transectos de $10 \mathrm{~m}$. De manera similar, se efectuó un gráfico de barras con las frecuencias absolutas (abundancias) para cada uno de los rangos de horas. Se realizó un análisis de varianza no paramétrico mediante una prueba de Kruskal Wallis. Se utilizaron los rangos de horas como criterios de clasificación bajo la estructura de tratamientos. La variable respuesta correspondió a las abundancias en dichos intervalos de horas.

Se seleccionaron los datos de las abundancias por género en cada uno de los 10 transectos y se expresaron binomialmente (presencia $=1$, ausencia $=0$ ) en una matriz. Con estos, se realizó un análisis de conglomerados para identificar agrupaciones naturales [7] de las abundancias de abejas en el cultivo. Esto se realizó, con el fin de analizar la similitud a través de la presencia de los cuatro géneros a lo largo del cultivo en los 10 transectos seleccionados. De acuerdo a [7] para este análisis, se aplicó el algoritmo de agrupamiento jerárquico UPGMA (Unweighted pair-group method using an arithmetic average) en función de la distancia euclidiana. Asimismo la media se uso como la medida de resumen.

Con los datos de $\mathrm{N}$ y $\mathrm{S}$, se realizó un análisis de diversidad con los índices de Shannon y Weiner (H'), dominancia de Simpson (Dsp) y riqueza de Margalef (DMg). Estos permitieron tener una aproximación general de la estructura de la comunidad polinizadora en el cultivo de estudio [8]. La estimación de los índices de diversidad se realizó con el programa ejecutable Divers franja 1993.

Se construyó con el programa InfoStat versión 2012 una curva de acumulación de especies con $\mathrm{S}$ y $\mathrm{N}$. Con el programa Estimates 7.5 [9] se realizaron cálculos del número de especies observadas y esperadas utilizando los estimadores Chao 1, ACE, ICE, Singletons (número de especies raras representadas por un único individuo en la muestra) y Doubletons (representadas exactamente por dos individuos) [Chao, 1984; Chao y Lee, 1992; Smith y van Belle, 1984; Colwell, 1997; Colwell y Coddington, 1994 por [10], además éstos índices son informativos ya que se estudian a escala de la comunidad, los cuales permiten tener una aproximación a su estructura [8]. Los números de los estimadores fueron graficados con las curvas de muestreo con Excel 2007.

\section{Análisis de la calidad del fruto}


Se llevó a cabo un Análisis de Componentes Principales (ACP). Para este se incluyó como variable de clasificación los géneros (3) Agapostemon, Apis y Melipona y la planta con polinización autógama (P. autógama). Las variables respuesta fueron: Peso $(\mathrm{g})$, diámetro ecuatorial y diámetro polar $(\mathrm{cm})$, siendo estas, variables morfométricas de interés económico. Asimismo, el número de semillas por fruto, fue una variable de interés ecológico. Con el fin de analizar el efecto de la polinización cruzada y la autógama sobre el peso del fruto, se realizó una prueba $\mathrm{T}$ para muestras independientes [7]. La variable de clasificación la conformó la polinización cruzada y la autógama. El peso representó la variable respuesta. Previo a la aplicación de la prueba, se evaluaron los supuestos de normalidad y homogeneidad de varianzas mediante un gráfico Q-Q plot y un diagrama de dispersión de Residuos vs Predichos respectivamente como se aplica en [7] (Figura 9, a y b). El análisis de normalidad se formalizó realizando una prueba de hipótesis sobre normalidad a través de una prueba de Shapiro wilks modificada por Gobindarajulu y Mahibbur, 1997 como se menciona en [7]. La homogeneidad de varianzas se corroboró con un análisis formal mediante la aplicación de una prueba $\mathrm{F}$ de igualdad de varianzas.

Para la aplicación de la prueba $\mathrm{T}$, se organizaron en una matriz los datos máximos. Estos se seleccionaron de la matriz de producción organizada previamente bajo un diseño factorial de una vía. Esta contenía los géneros y la polinización autógama como los niveles del factor de la variable de clasificación, asimismo, la variable respuesta fue el peso. Por lo tanto, al seleccionar los datos máximos, se buscó la equivalencia con el número de observaciones provenientes de la polinización autógama. Así, al estructurar la matriz de la prueba, se seleccionaron los datos de Agapostemon, Melipona y Apis, los cuales fueron útiles como criterio de clasificación de la prueba. De esta manera, los tres géneros constituyeron el nivel de factor llamado polinización cruzada que fueron contrastados con polinización autógama que representó el segundo nivel de factor. Para el análisis previo de normalidad y homogeneidad de varianzas, se utilizaron también los datos máximos del peso provenientes de la polinización por los géneros y los datos de la polinización autógama.

La prueba de Kruskal wallis, el análisis de conglomerados, Componentes Principales, y la validación de los supuestos al igual que la prueba $t$, se realizaron con el programa InfoStat versión 2012 [7].

Todos los análisis se ejecutaron bajo la plataforma Windows Xp.

\section{RESULTADOS}

A. Porcentaje de polinización

De los 100 individuos seleccionados, se marcaron en total 551 flores en antesis. De estas se obtuvieron 318 frutos, lo que índica que el estado actual de polinización (porcentaje de polinización) del cultivo es de 57.7\%. Por lo tanto, posiblemente el $42,3 \%$ que equivale a las flores que pese a presentar estado de antesis, no fructificaron siendo este uno de los problemas más comunes de este cultivo [1]. Esto indica que cerca al 50\% del cultivo no esta siendo aprovechado en términos de producción.

\section{B. Efectividad de visitantes florales}

En las zonas de mayor actividad pecoreadora identificadas, se obtuvo un total de 44 flores polinizadas por abejas. La observación ocho días después, indicó la presencia del fruto. 13 de estos equivalentes al $29.54 \%$ fueron abortados en su etapa inicial de desarrollo, posiblemente esto puede ser explicado por causas relacionadas con una polinización deficiente [1]; 16 frutos $(36.36 \%)$ presentaron algún tipo de enfermedad propias de este cultivo como 'la carachita' y la 'enfermedad de la mosca blanca' durante su desarrollo, siendo excluidos del análisis de producción. Finalmente, de los 44 frutos formados solo 15 de ellos (34.09\%) presentaron características de crecimiento y maduración normales. Independientemente del porcentaje de frutos abortados y enfermos, de 44 flores visitadas por abejas todas presentaron polinización efectiva.

\section{Análisis de abundancia}

La comunidad polinizadora está representada en tres géneros de la familia Apidae, estos fueron Melipona con una abundancia del 36\% al igual que el genero Apis. El menor porcentaje fue representado por Euglosa con el 7\% de la abundancia relativa. El género Agapostemon, fue el único representante de la familia Halictidae con el $21 \%$. La abundancia absoluta con 10 individuos de Apis y Melipona, indica que ambos géneros prevalecieron desde las 8:00am hasta las 4:00pm (Figura 3 y 4 ).

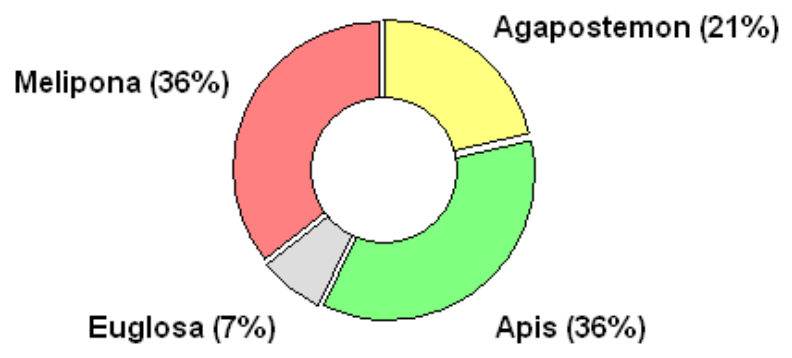

Figura 3. Abundancia relativa de los géneros de abejas presentes en el cultivo de tomate en condiciones de semi-invernadero.

Asimismo, la abundancia absoluta de los polinizadores por rangos de horas fue mayor en el rango de 8 a 10 am con 12 individuos. El horario con menor observación directa fue de 2$4 \mathrm{pm}$ con un individuo (Figura 4). Las abundancias absolutas decrecieron exponencialmente con aumento de la temperatura relativo al aumento de las horas. Esto ocurre al interior del cultivo, cuya temperatura promedio alcanzó $34^{\circ} \mathrm{C}$ en horas de la tarde durante los meses en los cuales se realizó el muestreo. La temperatura puede condicionar la actividad pecoriadora de 
las abejas y la efectividad en el proceso de polinización como ocurre en ápidos del género Bombus [3].

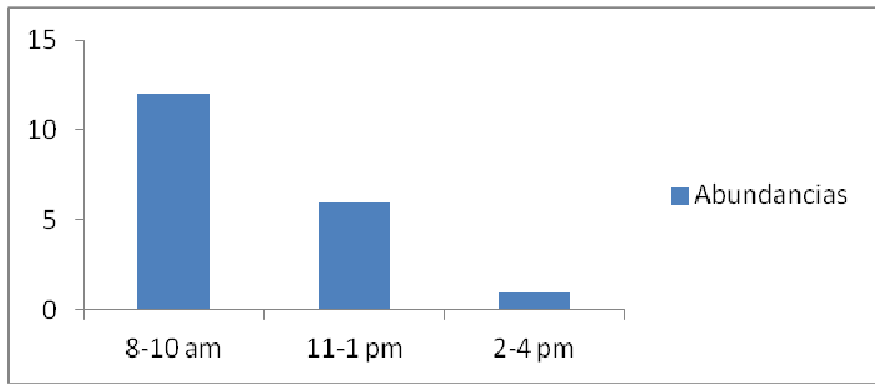

Figura 4. Abundancia absoluta de los polinizadores del cultivo de tomate en condiciones de semi-invernadero por rangos de horas.

El género con mayor número de individuos ocho correspondió a Melipona, las horas de mayor abundancia fue de 8 a $10 \mathrm{am}$. Apis estuvo presente en los tres rangos de horas, siendo este más abundante de $11 \mathrm{am}-1 \mathrm{pm}$. Euglossa presentó actividad de pecoreo sólo de $11 \mathrm{am}$ a $1 \mathrm{pm}$ con una abundancia baja (1 individuo) (Figura 5). Esto posiblemente se debe a que dicho género presenta polinización específica para algunas especies de la familia Orchidaceae, con caracteres del aparato lamedor - succionador modificados para la morfología floral de las orquídeas [11], aunque algunos euglosinos machos, ramonean algunas especias de la familia Solanaceae (Van den Eijnde $e t$ al., 1991 por [3].

El género Agapostemon y Melipona compartieron dos rangos de horas: de 8 a 10 am y de 11 a $1 \mathrm{pm}$. Por lo tanto su actividad de pecoreo es similar en relación a dichos rangos. Esto contrasta con que siendo ambas abejas silvestres, estas pertenecen a dos grupos diferentes; abejas solitarias para el caso de Agapostemon y abejas sociales representadas por Melipona [12].

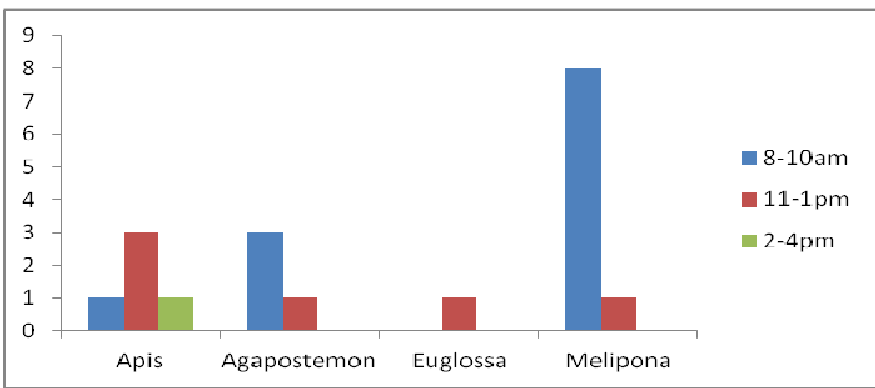

Figura 5. Abundancias absolutas entre géneros para cada uno de los rangos de horas en el cultivo de tomate.

El análisis de varianza mostró diferencias estadísticamente significativas $(\alpha=0.05)$ en la abundancia de la comunidad polinizadora entre rangos de horas con un $\mathrm{p}$-valor $=0.0387$. Por lo tanto la actividad de pecoreo de los polinizadores en el cultivo de tomate en relación su abundancia absoluta es mayor de 8 a 10 am con una media del ANOVA de 1.20 (Tabla 1, Figura 4).
Tabla 1. Análisis de varianza para las abundancias entre rangos de horas.

Prueba de Kruskal Wallis

\begin{tabular}{lllrlrcc} 
Variable & Hora & N & Medias & D.E. Medianas & H & p \\
\hline M.jundancias & $11-1 \mathrm{pm}$ & 10 & 0.60 & 0.70 & 0.50 & 5.01 & 0.0387 \\
M.bundancias & $2-4 \mathrm{pm}$ & 10 & 0.10 & 0.32 & 0.00 & & \\
M.bundancias & $8-10 \mathrm{am}$ & 10 & 1.20 & 1.32 & 1.00 & & \\
\hline
\end{tabular}

Mediante el análisis de similitud se conformó un grupo representado por los géneros Apis, Melipona y Agapostemon dada la medida de distancia euclidiana (vecino más cercano) en el plano unidimensional. Los géneros Apis y Melipona presentan el mismo registro de presencia en el cultivo de tomate, por lo tanto se aglomeran en un subgrupo. Pese a la baja abundancia de Euglosa, este estuvo presente en el cultivo de tomate teniendo en cuenta la especificidad de dicho género en las flores que visita. Por lo tanto, Euglosa se excluye del resto del grupo como se observa en la figura 6 .

Promedio (Average linkage) Distancia: (Euciiciea)

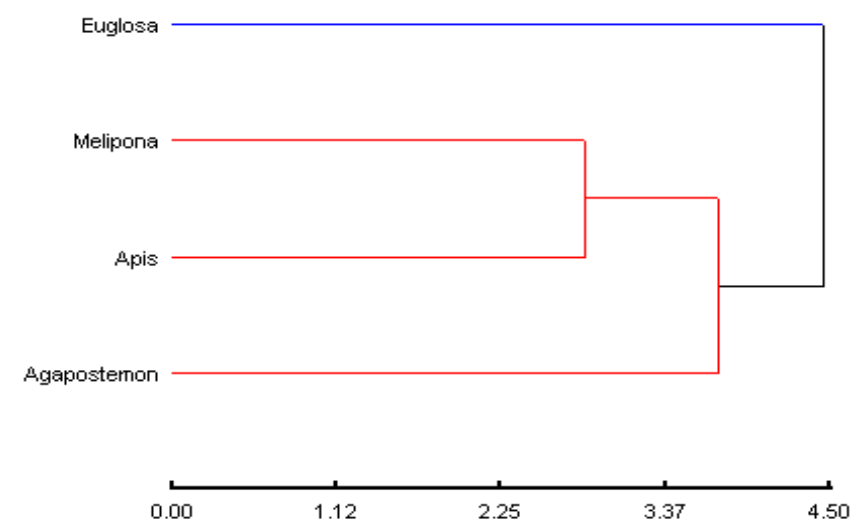

Figura 6. Análisis de conglomerados para la presencia/ausencia de los polinizadores del cultivo de tomate en condiciones de semiinvernadero.

\section{Análisis de calidad del fruto}

Las variables de importancia económica peso (Peso) y diámetro polar (Diámetro P) están altamente correlacionadas y muestran un tipo de asociación directa, perfecta $(\mathrm{R}=1)$, y estadísticamente significativa $(\mathrm{p}=0.0026)$ con un $\alpha=0.05$ (Tabla 2). Esta relación estadística, es una característica propia de la variedad analizada. La componente principal 1 y 2, explican el $99.9 \%$ de la varianza total $(\mathrm{CP} 1=78.3 \%$, $\mathrm{CP} 2=21.7 \%$ ) (Figura 7). Asimismo, el peso y el diámetro ecuatorial (Diámetro E) presentan una correlación directa y fuerte $(\mathrm{R}=0.99)$. Esta última variable, presentó el mismo valor del parámetro $\mathrm{R}$ asociado con la variable Diámetro (P) indicando alométricamente, que un aumento en una de las medidas implica el aumento de la otra. 
Tabla 2. Tabulación de las correlaciones, pruebas de hipótesis y valores propios del Análisis de componentes principales (Las tablas se obtuvieron con el programa InfoStat versión 2012).

\begin{tabular}{|c|c|c|c|c|}
\hline & Peso & Diámetro $\mathrm{E}$ & Diámetro P $\mathrm{N}$ & N semillas \\
\hline Peso & 1.00 & & & \\
\hline Diámetro $\mathrm{E}$ & 0.99 & 1.00 & & \\
\hline Diámetro $P$ & 1.00 & 0.99 & 1.00 & | \\
\hline $\mathrm{N}$ semillas & 0.38 & 0.23 & 0.37 & 1.00 \\
\hline
\end{tabular}

Matriz de correlación/Probabilidades

Peso Diámetro $\mathrm{E}$ Diámetro $\mathrm{P} \mathrm{N}$ semillas

\section{Peso}

Diámetro E 0.0127

Diámetro P 0.0026

N semillas 0.6211

0.0119

0.7713

0.6319

Autovalores

Lambda Valor Proporción Prop deum

$\begin{array}{rrrr}1 & 3.13 & 0.78 & 0.78 \\ 2 & 0.87 & 0.22 & 1.00 \\ 3 & 2.7 \mathrm{E}-03 & 6.7 \mathrm{E}-04 & 1.00\end{array}$

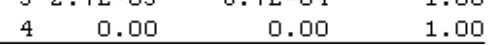

El número de semillas ( $\mathrm{N}$ semillas) como variable de interés ecológico, mostró una asociación débil $(\mathrm{R}=0.23)$ en relación a la variable diámetro ecuatorial. Esta asociación no fue estadísticamente significativa con un $\mathrm{p}=0.7713$. Asimismo, las asociaciones con el resto de variables fueron débiles Peso, $(\mathrm{R}=0.38)$ y Diámetro $\mathrm{P},(\mathrm{R}=0.37)$ (Tabla 2$)$. De este modo, se infiere que posiblemente el número de semillas para el fruto del tomate de la variedad analizada en este estudio, no parece afectar significativamente el peso como variable de interés económico para ésta. De este modo, en contraste con lo expuesto en la literatura aunque el peso no fue significativamente afectado, según [13], éste es mejorado por las abejas que a través de Buzz pollination o vibración por zumbido, permite que mayor número de granos de polen se deposite en el estigma de la flor aumentando el numero de semillas y formando un fruto de mayor peso. De esta manera la polinización a través de este mecanismo, se hace más efectiva [3].

El análisis y construcción de los vectores $\left(\xi_{1}\right.$ y $\left.\xi_{2}\right)$ dados por las CP1 y CP2, es el siguiente:

$\xi_{1}=0.56$ Peso +0.55 DiametroE +0.56 DiametroP +0.26 Nsemillas (1)

$\xi_{2}=-0.09$ Peso -0.25 DiametroE -0.10 DiametroP +0.96 Nsemillas

La primera componente, fue explicada principalmente por las variables Peso y Diámetro $\mathrm{P}$ con un peso o ponderado de 0.56 para cada una (Ec. 1, Tabla 3). El eigenvector o vector propio 0.96 asociado al $\mathrm{N}$ de semillas, explicó la mayor varianza de la segunda componente (CP2) (Ec. 2, Tabla 3).

Tabla 3. Tabulación de los eigenvectores de $\xi_{1}$ y $\xi_{2}$.

\begin{tabular}{lrr}
\multicolumn{3}{l}{ Autovectores } \\
Variables & e1 & \multicolumn{1}{c}{$\mathrm{e} 2$} \\
\hline Peso & 0.56 & -0.09 \\
Diámetro E & 0.55 & -0.25 \\
Diámetro P & 0.56 & -0.10 \\
N semillas & 0.26 & 0.96 \\
\hline
\end{tabular}

Las clasificaciones dadas por los géneros a través de las asociaciones con las características tanto económicas como ecológicas, mostraron que Agapostemon está más asociado con las variables de importancia económica de peso y el diámetro polar del fruto de tomate (Figura 7). Por lo tanto, dicho género es clave para la obtención de atributos de mejor calidad. En contraste, las plantas polinizadas mediante polinización autógama, no parecen estar relativamente asociadas con características de importancia tanto económica como ecológica (Figura 7 y 8). Por lo que se evidencia que Agapostemon, puede ser una opción apropiada para una polinización efectiva en pro del mejoramiento de la calidad del fruto desde el punto de vista de la producción como se observa en la figura 8 .

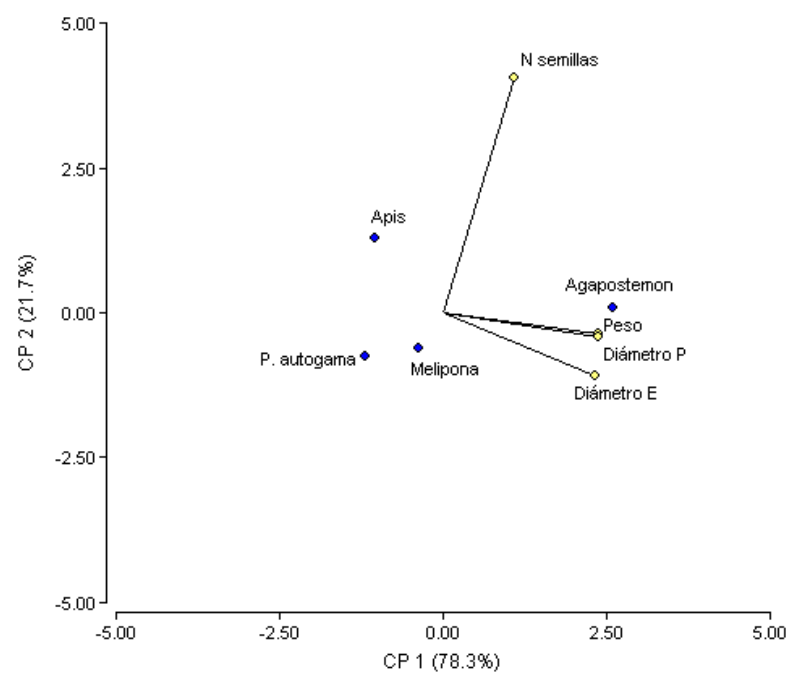

Figura 7. Análisis de Componentes Principales para los frutos producto de la polinización de los 3 géneros, después del periodo de seguimiento y alcanzada su madurez.

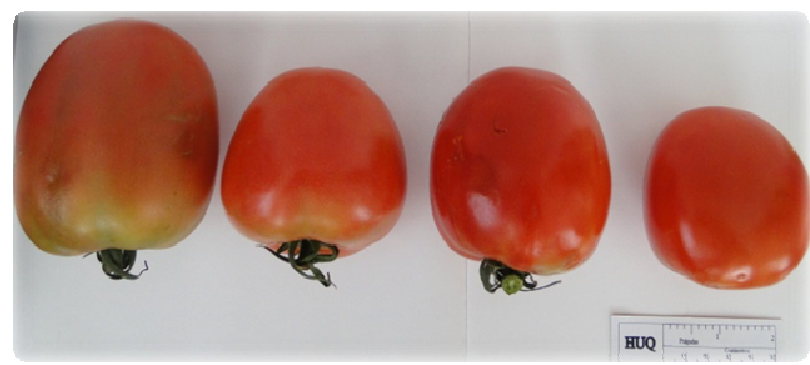

Figura 8. Secuencia de los mejores ejemplares de frutos obtenidos de la polinización y fruto control. Izquierda - derecha: 1) Fruto polinizado por Agapostemon, 2) Fruto de Melipona 3) Fruto de Apis y 4) fruto producto de polinización autógama (planta control).

\section{Análisis de los supuestos}

La distribución de los residuos (RDUOs) extraídos del análisis de varianza, muestran una dispersión cercana a la línea de tendencia de $45^{\circ}$, indicando que los errores se distribuyen normalmente como se observa en la figura 9a. De esta manera 
la prueba de hipótesis de normalidad con un p-valor igual a 0.4272 del estadístico de Shapiro wilks, indicó que no hay evidencia para rechazar el supuesto de normalidad (Tabla 4).
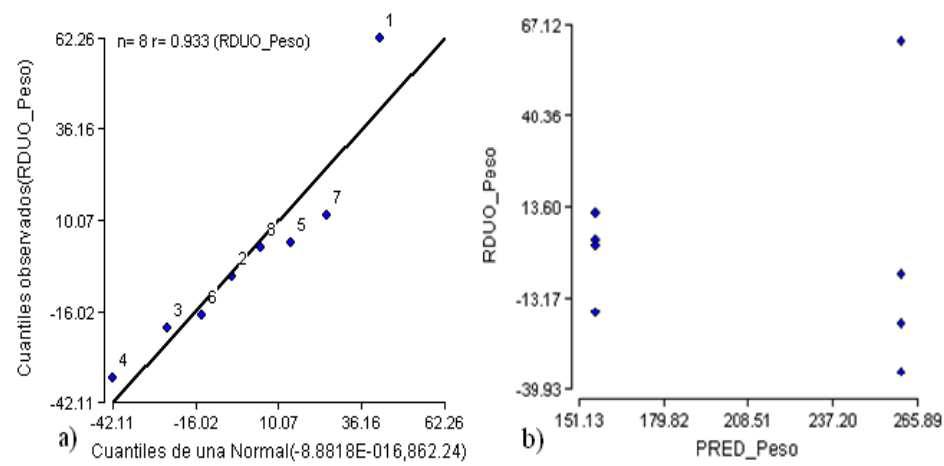

Figura 9. a) Q-Q plot que muestra que los errores se distribuyen normalmente. $\boldsymbol{b}$ ) Diagrama de Residuos vs predichos para la variable peso.

Tabla 4. Prueba de normalidad para el peso de los frutos de tomate analizados.

Shapiro-Wilks (modificado)

\begin{tabular}{lrrrrrr} 
Variable & n & Media & D.E. & \multicolumn{1}{c}{ W $^{*}$} & p (Unilateral D) \\
\hline RDUO Peso & 8 & 0.00 & 29.36 & 0.91 & 0.4272 \\
\hline
\end{tabular}

Simultáneamente en el análisis de homocedasticidad, la distribución estructurada de los puntos en el diagrama de dispersión, evidenció una posible falta de homogeneidad de varianzas como se observa en la figura $9 \mathrm{~b}$. Sin embargo, al aplicarse la prueba formal para probar la homocedasticidad, esta indicó evidentemente que las varianzas de las dos muestras son homogéneas con un $\mathrm{p}=0.0681$ de la prueba $\mathrm{F}$, el cual mostró que se acepta la hipótesis de homogeneidad de varianzas como se muestra en la tabla 5.

Tabla 5. Prueba de homocedasticidad para la variable peso de los frutos. La hipótesis de contraste corresponde a la igualdad de varianzas entre el peso de los frutos provenientes de la polinización autógama (grupo 1) y de polinización cruzada (grupo 2).

Prueba F para igualdad de varianzas

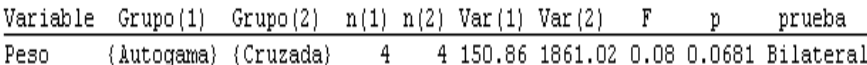

\section{Aplicación de la prueba $t$}

Una vez validados los supuestos de normalidad y homogeneidad de varianzas, con un $\alpha=0.05$, la prueba $t$ mostro diferencias estadísticamente significativas en el peso del fruto entre la polinización autógama y cruzada con un pvalor igual a 0.0035 (Tabla 6). Se infiere por tanto, que los frutos producto de polinización cruzada mostraron el mayor peso promedio (260.67). En contraste, el menor peso (media $=156.35)$ lo presentaron los frutos provenientes de polinización autógama. Esto, sugiere que la polinización cruzada llevada a cabo por las abejas es más efectiva, por lo que, frutos obtenidos a través de este mecanismo tienden a ser mas atractivos comercialmente. Asimismo el mayor peso promedio, demuestra una mejoría en la producción de tomate en relación al peso (Figura 8).

Tabla 6. Prueba para la comparación de medias entre frutos provenientes de ambos procesos de polinización.

Prueba I para muestras Independientes

\begin{tabular}{|c|c|c|c|c|c|c|c|}
\hline Polin & Peso & \{Lutogara\} & fCruzad & & & 156.35 & 260.67 \\
\hline & Media(: & 1) -Iedia (2) & LI(95) & LS(95) & $\mathrm{T}$ & p-valor & prueba \\
\hline & & -104.33 & -159.20 & -49.45 & -4.65 & 0.0035 & Bilateral \\
\hline
\end{tabular}

\section{E. Análisis de diversidad}

La interpretación de los estadísticos de diversidad, fueron basadas en los rangos según [14]. Por lo tanto, la aproximación a la estructura de la comunidad de polinizadores a través del análisis de los índices de diversidad, indicó que la riqueza de especies $\mathrm{DMg}=1.13677$ fue baja (Tabla 7). Pese a ello, este es un ponderado normal teniendo en cuenta que la unidad de estudio no es un ecosistema natural, por lo que se trata de una estructura (semi-invernadero) inmersa en un agroecosistema.

El estadístico de Simpson (Dsp) indicó que la dominancia de la comunidad analizada fue baja con un estimado de 0.25275 . La comunidad de polinizadores es heterogénea. En contraste, la comunidad fue más diversa que dominante, cuya diversidad en relación a Shannon con un $\mathrm{H}^{\prime}=1.25404$, fue baja. Así, relativo a la uniformidad de la muestra con un $\mathrm{E}=$ 0.90460 (Tabla 7).

Pese a los estimados de DMg, Dsp y H', el mecanismo de polinización cruzada a través de la estructura de la diversidad de polinizadores, tuvo efecto estadísticamente significativo sobre el peso (Tabla 6), la cual es una característica de interés comercial que mejora la producción.

Tabla 7. Índices de diversidad biológica a partir de la abundancia encontrada en los 10 transectos seleccionados.

Índices de diversidad.

\begin{tabular}{|cc}
\hline \multicolumn{2}{c}{ Índices de diversidad. } \\
\hline Abundancia (N) & 14 \\
\hline Riqueza de especies (S) & 4 \\
Uniformidad (E) & 0.90460 \\
Índice de Margalef (DMg) & 1.13677 \\
Índice de Simpson (Dsp) & 0.25275 \\
Índice de Shannon (H') & 1.25404 \\
\hline
\end{tabular}

F. Representatividad de la muestra

La curva de acumulación de las especies polinizadoras del cultivo construida a partir de los valores $\mathrm{N}$ a lo largo de los 10 transectos, indicó que al ser representativa la muestra, el 
esfuerzo de muestreo fue bueno con 20 individuos. Dicha curva tiene implícita la riqueza (valor S) dada por las especies presentes por unidad de área (transecto). La abundancia de los cuatro géneros determinados y muestreados a través los transectos fueron representativos de la estructura polinizadora del cultivo de tomate dado que la curva se mostró asintóticamente estable en X1 con 20 individuos (Figura 10).

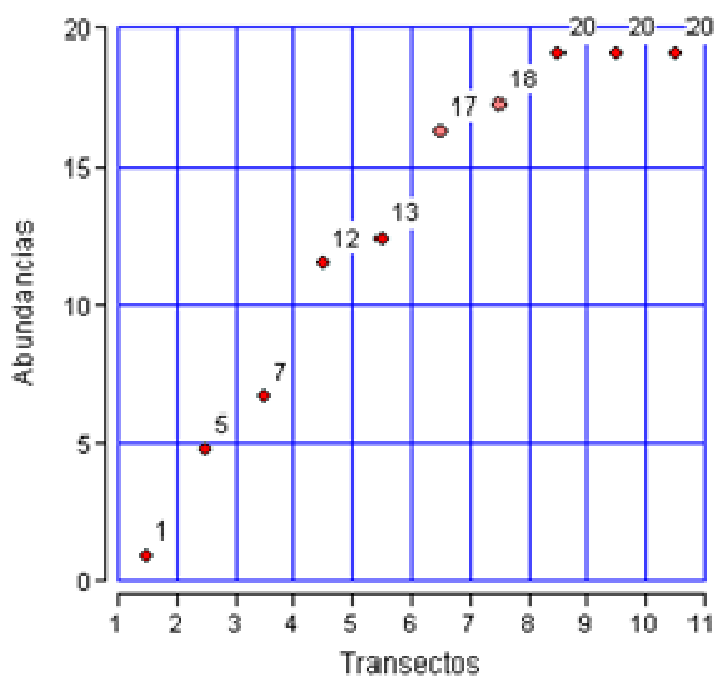

Figura 10. Curva de abundancias acumuladas a lo largo de los transectos muestreados del cultivo de tomate.

\section{Esfuerzo de muestreo}

En comparación al comportamiento asintótico de la curva de acumulación de abundancias (Figura 10), el muestreo fue bueno, ya que Chao 1 se comporta asintóticamente estable con los sobs (4) y ACE (4.4) (Figura 11, Tabla 8).

\begin{tabular}{rrcccc}
\hline sobs & ACE & $\begin{array}{l}\text { ICE } \\
\text { Mean }\end{array}$ & $\begin{array}{l}\text { chao } \\
\text { 1 }\end{array}$ & $\begin{array}{l}\text { Singletons } \\
\text { Mean }\end{array}$ & $\begin{array}{l}\text { Doubletons } \\
\text { Mean }\end{array}$ \\
\hline $\mathbf{1 , 3 9}$ & 2,19 & 2 & 2,19 & 1,35 & 0 \\
$\mathbf{2 , 3}$ & 3,77 & 4,26 & 3,26 & 1,81 & 0,48 \\
$\mathbf{2 , 8 6}$ & 4,73 & 5,27 & 3,78 & 1,78 & 0,88 \\
$\mathbf{3 , 2 7}$ & 5,05 & 5,31 & 4,04 & 1,41 & 1,23 \\
$\mathbf{3 , 4 6}$ & 4,65 & 5,24 & 3,86 & 1,31 & 1,14 \\
$\mathbf{3 , 6 4}$ & 4,5 & 4,43 & 3,95 & 0,98 & 1,05 \\
$\mathbf{3 , 7 3}$ & 4,3 & 4,38 & 3,88 & 0,93 & 0,73 \\
$\mathbf{3 , 8 2}$ & 4,26 & 4,32 & 3,88 & 0,88 & 0,47 \\
$\mathbf{3 , 9}$ & 4,27 & 4,32 & 3,9 & 0,89 & 0,25 \\
$\mathbf{4}$ & 4,4 & 4,48 & 4 & 1 & 0 \\
\hline
\end{tabular}

Tabla 8. Estimadores utilizados en la evaluación del esfuerzo de muestreo.

La tendencia de la curva de Chao 1 (Figura 11) con un estimado de 4 (Tabla 8) permite determinar la representatividad de los géneros. De este modo, el indicador sobs con 4 se acerca al número de esperados ACE con 4.4 (Figura 11, Tabla 8). El acercamiento de los valores de Chao 1 a los estimados de ACE permitió confirmar que el número de géneros encontrados fue apropiado, siendo esto un estimado que da importancia al rol de la diversidad en la polinización cruzada del cultivo. Los estimadores Singletons y Doubletons lo confirman, ya que en los primeros sitios de muestreo se presenta un mayor número de géneros raros (Figura 11), donde dichos géneros a medida que avanza el muestreo relativo a la estabilización de la curva óptima Chao 1, se hacen más comunes.

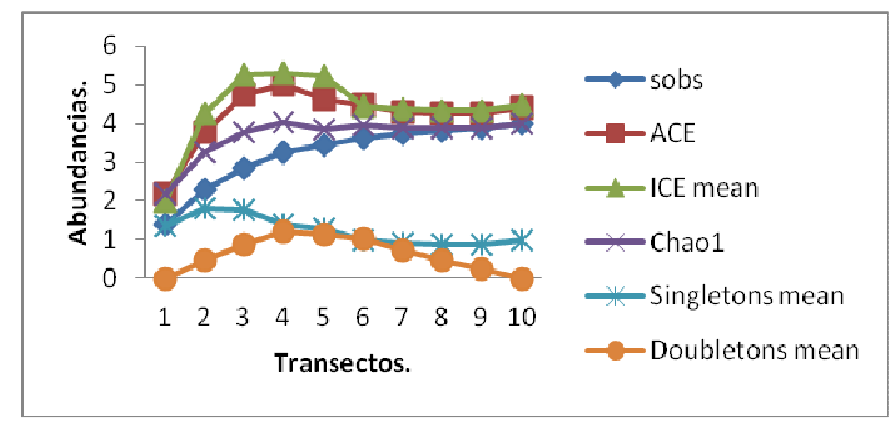

Figura 11. Curva de acumulación y estimaciones de la riqueza esperada en el cultivo de tomate utilizando estimadores y considerando las desviaciones estándar provenientes del proceso de aleatorización utilizando el programa Stimates 7.5.

\section{CONCLUSIONES}

- En el cultivo del tomate cerca del 50\% de las flores no están siendo aprovechadas en términos de producción.

- La diversidad de la comunidad polinizadora fue baja siendo ésta representada en su totalidad por abejas dentro de las cuales, el género Agapostemon es importante para la obtención de frutos de mejor calidad.

- La variable de interés económico peso, es favorecida por la polinización cruzada del cultivo dando importancia a la extensión del rol de las abejas en la polinización de este y otros cultivos.

\section{AGRADECIMIENTOS}

Al agricultor Fernando Antia del municipio de Viterbo Caldas, por permitirnos el acceso al cultivo para el desarrollo de la fase de campo.

\section{REFERENCIAS}

[1]. F. Nuez, El cultivo de Tomate: obra colectiva, edición ilustrada. Mundiprensa, 1999. p793.

[2]. J. Noreña, V. Rodríguez, A. Guzmán y M. Zapata, "El cultivo de tomate bajo invernadero (Licopersicon esculentum Mill)", Centro de Investigación la selva, CORPOICA., Antioquia, Colombia. Boletín técnico. 21, 2006.

[3]. J. Salinas, "Polinización del tomate (Lycopersicon esculentum Mill) en invernaderos en México". SEHUSA, S.A. de C.V, Volcán de Jorullo, México, 2000. 
[4]. J. Aldana, M. Almanza, D. Vecil, y D. Rodríguez, "Efectos de Bombus atratus (Hymenoptera: Apidae) sobre la productividad de tomate (Lycopersicon esculentum Mill) bajo invernadero en la Sabana de Bogotá, Colombia”, Revista de agronomía Colombiana, Vol. 25, No. 1, pp. 62-72, 2007.

[5]. S. Vásquez, H. Ballesteros, Y. Ortegón y U. Castro, "Polinización dirigida con Apis mellifera en un cultivo comercial de fresa", Reporte técnico. 2006.

[6]. M. Mendizábal, “Abejas. Manuales esenciales”. Buenos aires, Argentina. Editorial Albatros. 2005. p255.

[7]. J.A. Di Rienzo, F. Casanoves, M.G. Balzarini, L. Gonzalez, M. Tablada y C.W. Robledo. Software estadístico InfoStat. Grupo InfoStat, FCA, Universidad Nacional de Córdoba, Argentina. Versión 2012I. [Online] disponible en: http://www.infostat.com.ar.

[8]. H. Villareal, M. Alvarez, S. Córdoba, F. Escobar. G. Fagua, F. Gast, H. Mendoza, M. Ospina y A. M. Umaña. "Manual de métodos para el desarrollo de inventarios de biodiversidad". Programa de inventarios de Biodiversidad. Instituto de Investigaciones Alexander Von Humboldt. Bogotá, Colombia: Segunda edición, 2006. p236.

[9]. R. K. Colwell, 2005. EstimateS 7.5: Biodiversity Estimation. University of Connecticut. USA. [Online] disponible en: http://purl.oclc.org/estimates or http://viceroy.eeb.uconn.edu/estimates

[10]. C. Moreno, "Métodos para medir la biodiversidad". Centro de Investigaciones Biológicas. Universidad Autónoma del Estado de Hidalgo. México, 2001.

[11]. R. Dressler, "Euglossine bees (Hymenoptera: Apidae) of the Tambopata reserved zone, Madre de Dios, Perú," Revista Peruana de entomología, Vol. 27, pp75-79, 1985.

[12]. ICPA. 2010. Iniciativa Colombiana de Polinizadores. Capitulo Abejas. Universidad Nacional de Bogotá, Instituto de Investigación Alexander Von Humboldt. Bogotá, D.C. Colombia.

[13]. G. Nates-Parra, "Abejas silvestres y polinización”. Manejo Integrado de Plagas y Agroecología. Vol. 75, pp7-20. 2005.

[14]. [14] A. Ramírez, Ecología: Métodos de muestreo y análisis de poblaciones y comunidades.
Editorial Pontificia Universidad Javeriana, Bogotá. pp30, 55, 61. 2006 